\title{
THE CURVATURE OF A BÉZIER CONTROL POLYLINE
}

\author{
Bahadır Tantay ${ }^{1}$, Ferhat Taş ${ }^{2}$ \\ ${ }^{1}$ Ege University, Department of Mathematics, Izmir, 35100 Turkey, \\ bahadir.tantay@ege.edu.tr \\ 2 Istanbul University, Department of Mathematics, Istanbul, 34134 Turkey \\ tasf@istanbul.edu.tr
}

\begin{abstract}
The role of differential geometry in describing a curve can not be denied. The differential forms defined for Bézier curves which are widely used in computer aided geometric design, plays a significant role in classification and image processing of curves. For this reason, the definitions such as Serret-Frenet frame, curvature and torsion which are described for Bézier curves are very important in computer aided geometric design. In this paper, in addition to these definitions we have also defined a new classification by applying angular curvature used for planar curves in computational geometry to Bézier control polygon.
\end{abstract}

Key Words- Bézier curves, blossom, line curvature.

\section{INTRODUCTION}

\subsection{Representation of curves}

Among the representations used for curves parametric representation is the most commonly used one owing to advantages in representing curves. In the parametric method a curve is represented as a function of one parameter.

$$
\alpha(\mathrm{t}): \mathrm{I} \subset \mathbf{R} \rightarrow \mathbf{R}^{\mathrm{n}}, \mathrm{n}=2,3, \ldots
$$

We look for class of functions which is as simple as possible and yet diverse enough to represent a wide variety of curves. Polynomial functions to large extent, satisfy this requirement. A general polynomial function is shown below:

$$
\alpha(\mathrm{t})=\sum_{\mathrm{i}=0}^{\mathrm{n}} \mathrm{p}_{\mathrm{i}} \mathrm{t}^{\mathrm{i}}
$$

where $\mathrm{n}$ is the degree of the polynomial [1].

The variety of curves that can be obtained by using polynomials depends on the maximum allowed degree. The higher the degree, the greater variety of shapes one can represent. For example, to define a curve with $\mathrm{n}$ wiggles, we need a polynomial of degree $n+1$. But higher degrees result in some problems. The higher the degree of a curve, the less controllable it is, in a sense that small changes in coefficients are likely to result in large changes in the shape of the curve [1].

Consider the functional case only. Degree 1 polynomials can only be used to represent straight lines; degree 2 curves are parabolas in non-degenerate cases. The parabola does not have a point of inflection, where the curve turns from being concave to convex or vice-versa. Cubic curves may have inflection points [1].

To represent arbitrarily complex curves one usually uses piecewise polynomials, stitching together many polynomial pieces.

To represent the curves in space we commonly use: 


$$
\alpha(t)=p_{0}+p_{1} t+p_{2} t^{2}+p_{3} t^{3}
$$

where $p_{i}$ 's are points in three dimensional space [1].

\section{BÉZIER CURVES}

\subsection{Definition}

Generally, a Bézier curve is a polynomial curve with degree $n$ and shown in vector form as:

$$
\mathrm{B}(\mathrm{t})=\sum_{\mathrm{i}=0}^{\mathrm{n}} \mathrm{b}_{\mathrm{i}} \mathrm{B}_{\mathrm{i}}^{\mathrm{n}}(\mathrm{t}), \quad \mathrm{t} \in[0,1]
$$

where

$$
B_{i}^{n}(t)=\left\{\begin{array}{cl}
\frac{n !}{i !(n-i) !}(1-t)^{n-i} t^{i} & , 0 \leq i \leq n \\
0 & , \text { otherwise }
\end{array}\right.
$$

is Bernstein bases function and $\frac{n !}{i !(n-i) !}$ is binomial coefficient [1].

\subsection{Properties of Bézier curves}

a) Interpolation: One can easily see that $\mathrm{B}(0)=\mathrm{b}_{0}$ and $\mathrm{B}(1)=\mathrm{b}_{\mathrm{n}}[1]$.

b) Affine Invariance: This property can be easily verified by considering an affine map $\varphi(x)=A x+v$ where $A$ is a nxn matrix and $\mathrm{v}$ is in $\mathrm{R}^{n}$ [1].

$$
\varphi\left(\sum_{\mathrm{i}=0}^{\mathrm{n}} \mathrm{b}_{\mathrm{i}} \mathrm{B}_{\mathrm{i}}^{\mathrm{n}}(\mathrm{t})\right)=\mathrm{A} \sum_{\mathrm{i}=0}^{\mathrm{n}} \mathrm{b}_{\mathrm{i}} \mathrm{B}_{\mathrm{i}}^{\mathrm{n}}(\mathrm{t})+\mathrm{v}=\sum_{\mathrm{i}=0}^{\mathrm{n}}\left(\mathrm{A} \mathrm{b}_{\mathrm{i}}+\mathrm{v}\right) \mathrm{B}_{\mathrm{i}}^{\mathrm{n}}(\mathrm{t})=\sum_{\mathrm{i}=0}^{\mathrm{n}} \varphi\left(\mathrm{b}_{\mathrm{i}}\right) \mathrm{B}_{\mathrm{i}}^{\mathrm{n}}(\mathrm{t})
$$

c) Tangent to the curve at point $\mathbf{b}_{\mathbf{0}}$ is the vector $\overrightarrow{\mathrm{b}_{0} \mathrm{~b}_{1}}$ and tangent to the curve at $\mathbf{b}_{3}$ is $\overrightarrow{b_{2} b_{3}}$ This property can be used to test whether two Bézier curves are joined smoothly [1].

d) Convex Hull: It is clear that $B_{i}^{n}(t) \geq 0$ and $\sum B_{i}^{n}(t)=1$, for $t \in[0,1]$. This means that all points on the Bézier curve lie inside the convex hull of points $\mathrm{b}_{0}, \mathrm{~b}_{1}, \ldots, \mathrm{b}_{\mathrm{n}}[1]$.

\section{POLAR FORMS AND BLOSSOMING}

Polar form of a polynomial of $\alpha(\mathrm{t}): \mathbf{R} \rightarrow \mathbf{R}^{\mathrm{n}}$ of degree $\mathrm{n}$ is a multi-affine symmetric function $\alpha\left(\mathrm{t}_{1}, \mathrm{t}_{2}, \ldots, \mathrm{t}_{\mathrm{n}}\right): \mathbf{R}^{\mathrm{n}} \rightarrow \mathbf{R}$ such that [1]

$$
\alpha(\underbrace{\mathrm{t}, \mathrm{t}, \ldots, \mathrm{t}}_{\mathrm{n}})=\alpha(\mathrm{t})
$$

\subsection{Properties of polar forms}

a) A function $f\left(t_{1}, t_{2}, \ldots, t_{n}\right)$ is symmetric if the value of the function is the same for any permutation of the arguments $t_{1}, t_{2}, \ldots, t_{n}[1]$. 
b) A function $f\left(t_{1}, t_{2}, \ldots, t_{n}\right)$ is multi-affine if for any arguments $\mathrm{t}_{1}, \ldots, \mathrm{t}_{\mathrm{j}}^{0}, \mathrm{t}_{\mathrm{j}}^{1}, \ldots, \mathrm{t}_{\mathrm{n}}$ and any $\mathrm{s}$;

$f\left(t_{1}, \ldots, s t_{j}^{0}+(1-s) t_{j}^{1}, \ldots, t_{n}\right)=s f\left(t_{1}, \ldots, t_{j}^{0}, t_{j+1}, \ldots, t_{n}\right)+(1-s) f\left(t_{1}, \ldots, t_{j}^{1}, \ldots, t_{n}\right)[1]$.

For any polynomial, there exists a unique symmetric and multi-affine polar form. For example, a straight line given like this form: $F=a x+b$. Then if we polarize this first order polynomial we obtain $\mathrm{f}=\mathrm{at}_{0}+\mathrm{b}$. The general quadratic polynomial function form is $G=a x^{2}+b x+c$. If we polarize it, then we obtain that $g=a t_{0} t_{1}+b\left(t_{0}+\right.$ $\left.\mathrm{t}_{1}\right) / 2+\mathrm{c}$. Also, the elementary symmetric functions of the cubic polynomial function are [1]

$$
\left[\begin{array}{llll}
1 & \frac{\mathrm{t}_{0}+\mathrm{t}_{1}+\mathrm{t}_{2}}{3} & \frac{\mathrm{t}_{0} \mathrm{t}_{1}+\mathrm{t}_{1} \mathrm{t}_{2}+\mathrm{t}_{2} \mathrm{t}_{0}}{3} & \mathrm{t}_{0} \mathrm{t}_{1} \mathrm{t}_{2}
\end{array}\right]
$$

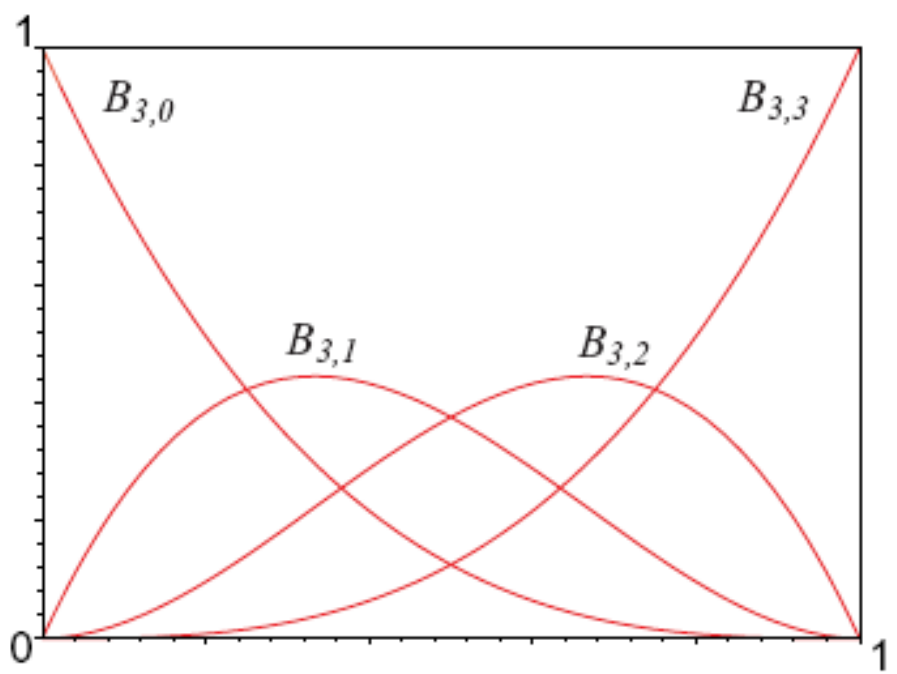

Figure 1: Bernstein basis function for $\mathrm{n}=3$ 

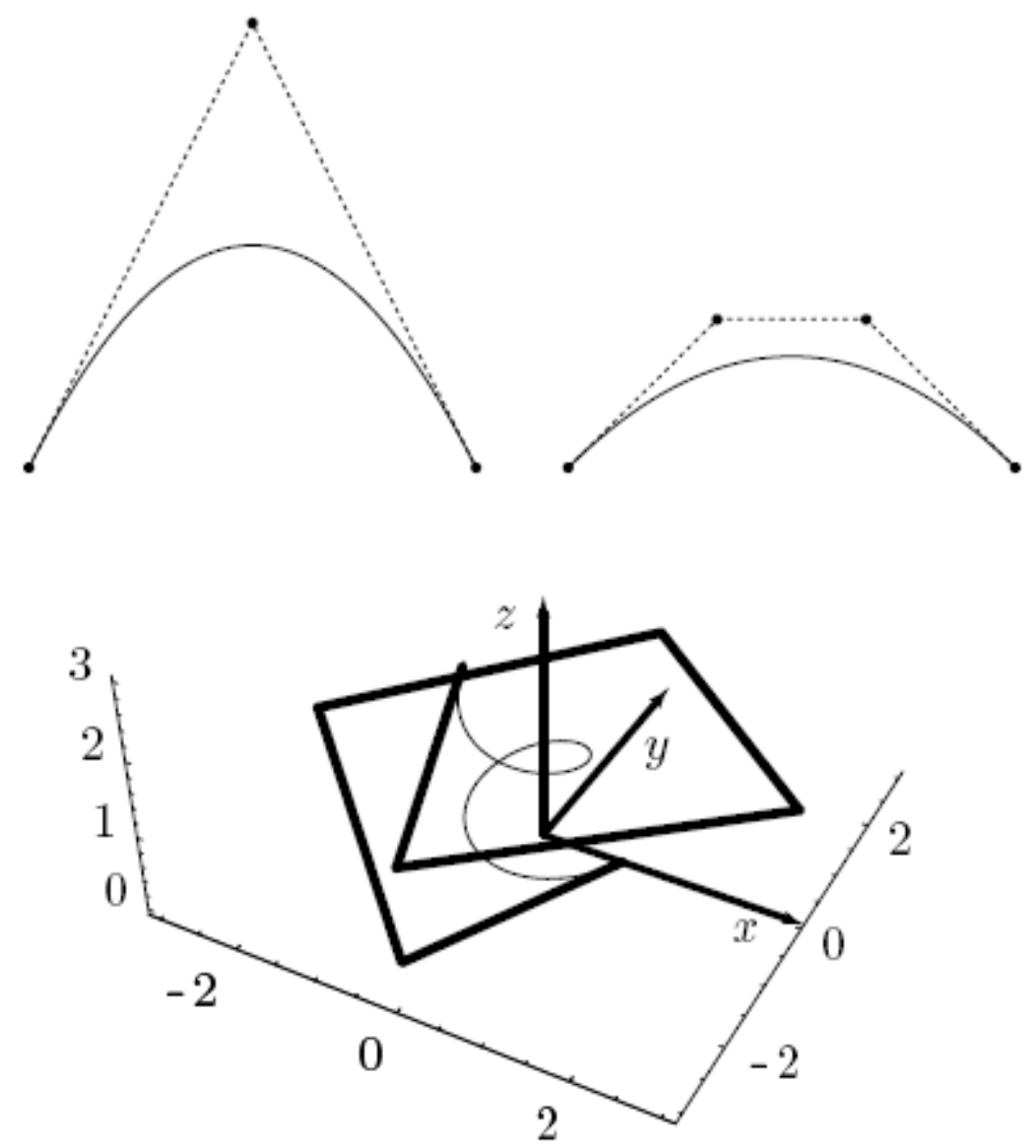

Figure 2: Bézier curves for $n=2,3$

\subsection{Example}

Suppose we want to construct a parabola, $y=x^{2}$ with the simple parameterization of,

$\mathrm{F}_{0}(\mathrm{t})=\mathrm{t}$,

$F_{1}(t)=t^{2}$

First we find the polar forms of Fs.

$\mathrm{f}_{0}\left(\mathrm{t}_{0}, \mathrm{t}_{1}\right)=\left(\mathrm{t}_{0}+\mathrm{t}_{1}\right) / 2$,

$\mathrm{f}_{1}\left(\mathrm{t}_{0}, \mathrm{t}_{1}\right)=\mathrm{t}_{0} \mathrm{t}_{1}$

Next, we find the control points defining the 32 -affine mappings.

$\mathrm{b}_{0}=\left(\mathrm{f}_{0}(0,0), \mathrm{f}_{1}(0,0)\right)=(0,0)$,

$b_{1}=\left(f_{0}(0,1), f_{1}(0,1)\right)=(1 / 2,0)$,

$\mathrm{b}_{2}=\left(\mathrm{f}_{0}(1,1), \mathrm{f}_{1}(1,1)\right)=(1,1)$

Finally we get the constructive parabola as in Figure 3 [2]. 




Figure 3 : Quadratic curve and its control points.

\section{THE CURVATURE OF A POLYGONAL LINE}

Consider a convex $\mathrm{n}$-sided polygon or $\mathrm{n}$-gon. From interior point $\mathrm{z}$ we draw lines connecting $\mathrm{z}$ to the vertices of the $\mathrm{n}$-gon. Since the $\mathrm{n}$-gon is convex, these connecting lines are all interior and they divide the $\mathrm{n}$-gon into $\mathrm{n}$ triangles, as indicated schematically in Figure 4 [5].

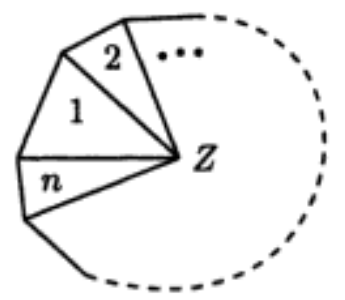

Figure 4: n-gon.

Let the angles of the $i$ th triangle be $\alpha_{i}, \beta_{i}, \gamma_{i}$ in clockwise order around the triangle such that $\gamma_{i}$ is the vertex at $\mathrm{z}$. An (interior) angle of the n-gon is of the form $\alpha_{i+1}+\beta_{i}$, except for the last, which is $\alpha_{1}+\beta_{n}$. Hence the sum of the angles of the $n$-gon is $\sum_{\mathrm{i}=1}^{\mathrm{n}}\left(\alpha_{\mathrm{i}}+\beta_{\mathrm{i}}\right)$. Since the sum of the angles $\gamma_{\mathrm{i}}$ at $\mathrm{z}$ is $2 \pi$, we can write sum of the angles of the $n$-gon $=\sum\left(\alpha_{i}+\beta_{i}+\gamma_{i}\right)-2 \pi$ [5].

If $\delta_{i}$ denotes the effect of the $i$ th triangle, we have $\alpha_{i}+\beta_{i}+\gamma_{i}=\pi-\delta_{i}$. We therefore make the following definition. 


\subsection{Definition}

The angular defect of the n-gon is (n-2) $\pi$ - the sum of its angles [5]. Let $G$ be a polygonal line and $\mathrm{p}$ one of its interior vertices. Let $\angle(\mathrm{p}) \in[0, \pi)$ be the turning angle between the two consecutive edges $\mathrm{k}_{1}$ and $\mathrm{k}_{2}$ adjacent to $\mathrm{p}$, of length $\mathrm{N}_{1}$ and $\mathrm{N}_{2}$. The curvature $c(p)$ of $G$ at $p$ is defined by the angular defect

$$
\mathrm{c}(\mathrm{p})=\frac{2(\pi-\angle(\mathrm{p}))}{\mathrm{N}_{1}+\mathrm{N}_{2}}
$$

So, $c(p)$ vanishes when the two edges are collinear [3].

\section{THE CURVATURE OF A BÉZIER CONTROL POLYLINE}

Let a Bézier curve with degree $\mathrm{n}$ is determined by $\left\{\mathrm{b}_{0}, \mathrm{~b}_{1}, \ldots, \mathrm{b}_{\mathrm{n}}\right\}(\mathrm{n}>1)$ control vertices. So the set which includes the control vertices can be defined as an oriented simplex. Each consecutive pair of points determine an edge. So we can define the curvature of a Bézier control polyline at each pair of consecutive edges based on definition in 4 . As in the general geometry formula

$$
\arccos \left(\frac{<\overrightarrow{\mathrm{u}}, \overrightarrow{\mathrm{v}}>}{\|\overrightarrow{\mathrm{u}}\| \cdot\|\overrightarrow{\mathrm{v}}\|}\right)=\theta
$$

where $\overrightarrow{\mathrm{u}}$ and $\overrightarrow{\mathrm{v}}$ are any vectors in $\mathrm{E}^{\mathrm{n}}$ without their magnitude is zero. So each consecutive control vertex determines a vector and an angle in $\mathrm{E}^{\mathrm{n}}$.

Let $b_{i-1}, b_{i}, b_{i+1}$ be three consecutive control points. Angular curvature at $b_{i}$ is determined by

$$
\mathrm{A}\left(\mathrm{b}_{\mathrm{i}}\right)=\frac{2\left(\pi-\angle\left(\mathrm{b}_{\mathrm{i}-1} \mathrm{~b}_{\mathrm{i}}\right)\left(\mathrm{b}_{\mathrm{i}} \mathrm{b}_{\mathrm{i}+1}\right)\right)}{\left\|\mathrm{b}_{\mathrm{i}-1} \mathrm{~b}_{\mathrm{i}}\right\|+\left\|\mathrm{b}_{\mathrm{i}} \mathrm{b}_{\mathrm{i}+1}\right\|}
$$

\subsection{Example}

Consider a quadratic Bézier curve given with control points $b_{0}=(0,0), b_{1}=(0,5)$ ve $b_{2}=(6,7)$. If we use the Bernstein basis form;

$$
\alpha(t)=(1-t)^{2} b_{0}+(1-t) t b_{1}+t^{2} b_{2}
$$

then equation of the curve is

$$
\alpha(t)=\left(6 t^{2}, 2 t^{2}+5 t\right)
$$

Approximate values of the curvature of the curve at $t=0$ and $t=1$ are 0.48 and 0.017 , respectively. and $b_{2}$.

On the other hand, we can compute the curvature of the Bézier polyline at $b_{0}, b_{1}$

$$
\begin{aligned}
& \mathrm{A}\left(\mathrm{b}_{0}\right) \cong 3.315 \\
& \mathrm{~A}\left(\mathrm{~b}_{1}\right) \cong 1.384 \\
& \mathrm{~A}\left(\mathrm{~b}_{2}\right) \cong 3.031
\end{aligned}
$$

$b_{1}$ is not on the curve and if we wish to change it; 
For example let $b_{1}=(1,3)$. Then the equation of the curve is $\beta(t)=\left(5 t^{2}+t, 4 t^{2}+3 t\right)$. Approximate values of the curvature of the curve at $A\left(b_{1}\right) \cong 1.641$. So the curvature of the Bézier polyline has changed by changing the control points. Also the curvature of the curve has changed. Approximate values of the curvature of the curve at $\mathrm{t}=0$ and $\mathrm{t}=1$ are 0.695 and 0.005 , respectively. Figure 5 shows the difference between $\alpha(t)$ and $\beta(t)$.

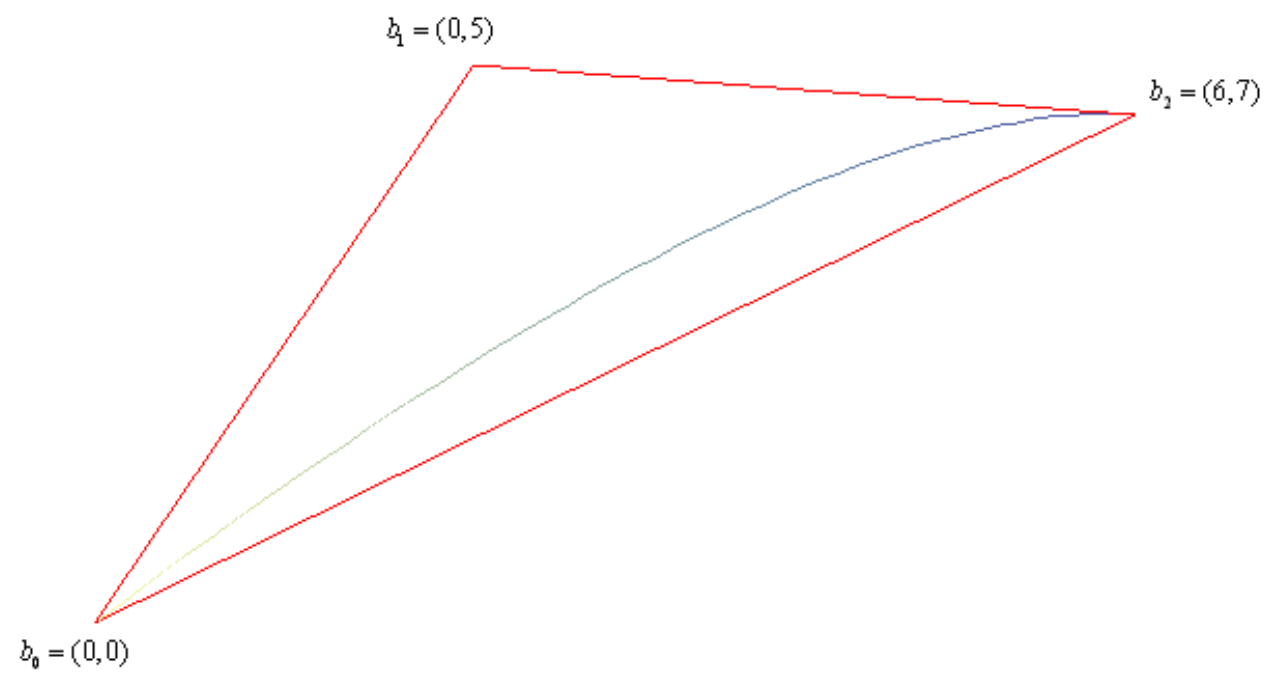

(a)

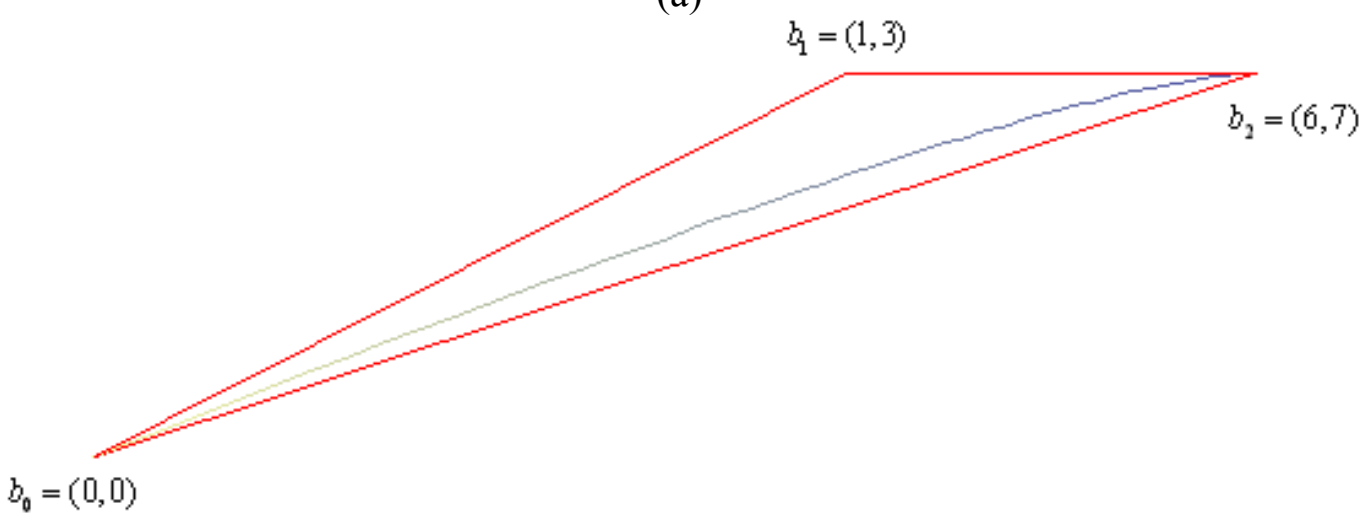

(b)

Figure 5: (a) $\alpha(\mathrm{t})$ and its Bézier control polygon. (b): $\beta(\mathrm{t})$ and its Bézier control polygon.

\section{CURVATURE OF THE BÉZIER POLYLINE OF A PARAMETRIC CURVE WITH DEGREE N}

Consider a parametric curve with degree $n$ represented by $\alpha(t)=\sum_{i=0}^{n} p_{i} t^{i}$. Its polar form is

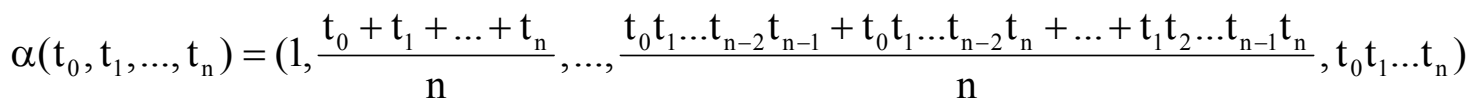


So the curve has $n+1$ control points $b_{0}, b_{1}, \ldots, b_{n+1}$, and we can determine the angular curvature of the curve by using these control points.

\section{Example 3}

Let the curve be $\alpha(t)=\left(3 t-t^{3}, 3 t^{2}, 3 t+t^{3}\right)$. Since the curve is cubic, there are four control points. As we explained in section 3 we find the control points via using the blossoming as

$\mathrm{b}_{0}=(0,0,0), \mathrm{b}_{1}=(1,0,1), \mathrm{b}_{2}=(2,1,2)$ and $\mathrm{b}_{3}=(2,3,4)$.

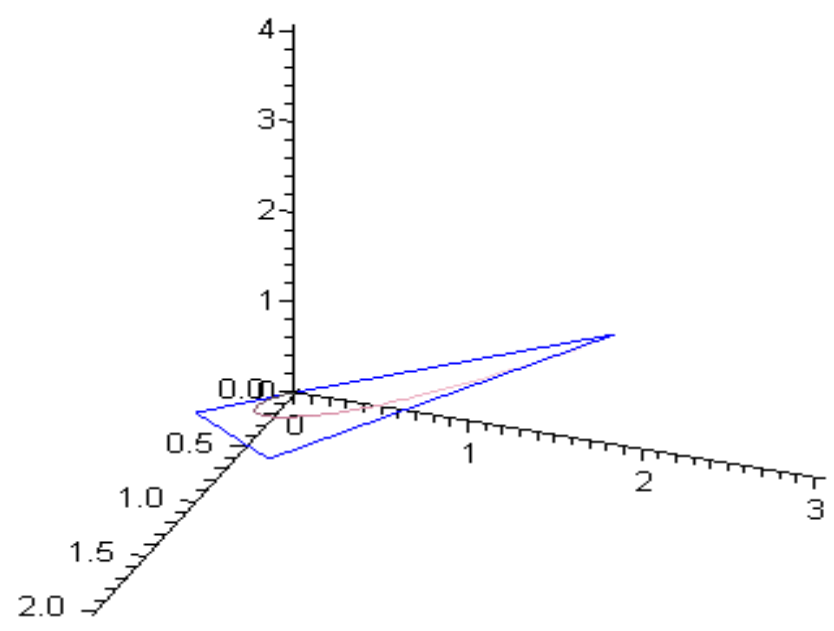

Figure 6: The curve $\alpha(t)$ and its control polygon

The curvature of the curve at $b_{0}$ and $b_{3}$ are $1 / 3$ and $1 / 12$, respectively. The approximate value of the curvatures of the Bézier control polyline at $b_{1}$ and $b_{2}$ are 4.703 and 3.418, respectively.

\section{CONNECTION BETWEEN CURVATURE AND CURVATURE}

We know that the curvature of a Bézier curve is formulated with its control points as follow [4]:

$$
\mathrm{C}_{\mathrm{E}}=\frac{2}{3} \frac{\left|\left(\overrightarrow{\mathrm{b}_{0} \mathrm{~b}_{1}}\right) \times\left(\overrightarrow{\mathrm{b}_{1} \mathrm{~b}_{2}}\right)\right|}{\left|\overrightarrow{\mathrm{b}_{0} \mathrm{~b}_{1}}\right|^{3}}
$$

Also we have the curvature of a Bézier control polyline of the corresponding curve with its control points as:

$$
A_{E}=2 \frac{\left.\pi-\arcsin \left(\frac{\left.\mid \overrightarrow{b_{0} b_{1}}\right) \times\left(\overrightarrow{b_{1} b_{2}}\right) \mid}{\left|\overrightarrow{b_{0} b_{1}}\right|\left|\overrightarrow{b_{1} b_{2}}\right|}\right)\right]}{\left|\overrightarrow{b_{0} b_{1}}\right|+\left|\overrightarrow{b_{1} b_{2}}\right|}
$$




$$
\Rightarrow C_{E}=\frac{2}{3} \frac{\left|\overrightarrow{b_{1} b_{2}}\right|}{\left|\overrightarrow{b_{0} b_{1}}\right|^{2}} \sin \left[\pi-\left(\frac{\left|\overrightarrow{b_{0} b_{1}}\right|+\left|\overrightarrow{b_{1} b_{2}}\right|}{2}\right) \cdot A_{E}\right]
$$

So we have a connection between angular curvature and curvature.

\section{CONNECTION BETWEEN ANGULAR CURVATURE AND TORSION}

We know that the torsion of a Bézier curve is formulated with its control points as follow [4]:

$$
B_{E}=\frac{1}{3} \frac{\left[\left(\overrightarrow{b_{1}-b_{0}}\right),\left(\overrightarrow{b_{2}-b_{1}}\right),\left(\overrightarrow{b_{3}-b_{2}}\right)\right]}{\left|\left(\overrightarrow{b_{1}-b_{0}}\right) \times\left(\overrightarrow{b_{2}-b_{1}}\right)\right|^{2}}=\frac{1}{3} \frac{\left[\left(\overrightarrow{b_{0} b_{1}}\right),\left(\overrightarrow{b_{1} b_{2}}\right),\left(\overrightarrow{b_{2} b_{3}}\right)\right]}{\mid\left(\overrightarrow{b_{0} b_{1}}\right) \times\left(\overrightarrow{b_{1} b_{2}}\right)^{2}}
$$

Also we have curvature of a Bézier control polyline as mentioned above, then we have;

$$
B_{E}=\frac{1}{3} \frac{\left[\left(\overrightarrow{b_{0} b_{1}}\right),\left(\overrightarrow{b_{1} b_{2}}\right),\left(\overrightarrow{b_{2} b_{3}}\right)\right]}{\left|\overrightarrow{b_{0} b_{1}}\right|\left|\overrightarrow{b_{1} b_{2}}\right| \sin \left(\pi-G_{E} \cdot \frac{\left|\overrightarrow{b_{0} b_{1}}\right|+\left|\overrightarrow{b_{1} b_{2}}\right|}{2} \mid\right)^{2}}
$$

Therefore, we have a connection between curvature of the Bézier control polyline and torsion.

\section{CONCLUSION}

For a Bézier curve to obtain the control points which allow us to make some changes on the curve given in its parametric form, we can use the blossoming method. Bézier curves are very important in the manufacturing of automobile and ship parts. Curves can be used in computer aided geometric design in a similar way that they are used in differential geometry. While in classical differential geometry, a curve is defined by using its curvature, here we do it by using control points. This idea resulted using them in the Bézier curves and their control polygon. Finally, we obtain the characterization of the curves.

\section{REFERENCES}

1. Zorin, D., Bézier Curves and B-splines, Blossoming, Geometric Modeling, New York University ,G22.3033-002: Topics in Computer Graphics: Lecture \#2, 2002.

2. Chen, X.., An Introduction to Blossoming of Bsplines, School of Computing, University of Utah, Salt Lake City, UT 84112.

3. Boissonnat, J.D., Teillaud,M., Effective Computational Geometry for Curves and Surfaces, Springer, 2006.

4. Marsh,D., Applied Geometry for Computer Graphics and CAD, Springer, 2005.

5. Ramsay, A., Richtmyer, R.D., Introduction to Hyperbolic Geometry, Springer, 1995. 